\title{
Novel high-pressure phases of AIN: a first-principles study
}

Chao Liu, Meng Hu, Kun Luo, Lin Cui, Dongli Yu, Zhisheng Zhao, Julong He*

State Key Laboratory of Metastable Materials Science and Technology, Yanshan University, Qinhuangdao 066004, China

E-mail addresses:hjl@ysu.edu.cn (Julong He);

\begin{abstract}
Four novel AIN phases with low density and high hardness are predicted by using a evolutionary methodology on structural search. All four phases are thermodynamically more favourable than the rock salt structure of AIN at ambient pressure and will be transformed to rock salt AIN at certain pressure. All these phases are mechanically and dynamically stable by checking the independent elastic constants and phonon dispersion spectra. The calculated mechanical properties reveal that the proposed AIN phases are hard, and the Vickers hardness of the AIN phases ranges from 13.2 GPa to $15.2 \mathrm{GPa}$. The calculated band structures of the novel AIN phases reveal that these AIN phases possess semiconductive properties with wide direct band gaps at $\mathrm{G}$ points, and the gaps range from $3.627 \mathrm{eV}$ to $3.927 \mathrm{eV}$.
\end{abstract}

\section{Introduction}

Aluminium nitride (AIN) has been attracted extensive attention on theoretical and experimental studies due to its importance of scientific research and industrial application. At ambient conditions, AIN exists as a closely packed hexagonal wurtzite structure (wzAIN), which has a widely applications, including cutting and machining tools, semiconductor devices, and field-emission devices because of its excellent chemical and physical properties, such as high thermal conductivity [1, 2], high melting point [3], large bulk modulus [4-6], good hardness [7-9], and corrosion resistance [10], large band gap [11].

The materials' chemical and physical properties are related to their structures. Scientists have been searching for novel allotropes of AIN, which may have other important applications. A zinc blende structure AIN (zb-AIN) has been synthesised by a solid-state reaction [12]. Scientists have acquired a rock salt structure AIN (rsAIN) transformed from wz-AIN by utilizing high pressure technology [13-16]. This phase transition has been reported to begin at 14-16.6 GPa [13-16]. Theoretical studies on AIN structures are +underway. In 1973, van Vechten predicted a transition to a $\beta$-Sn structure (for the diatomic equivalent of the $\beta$-Sn structure of binary compound; this structure was also named $d-\beta-S n$ and its space group is $1 \overline{4} \mathrm{~m} 2$ $[17,18])$ at $90 \mathrm{GPa}[19]$, whereas no phase transitions to $\beta$-Sn were observed $[15,20]$. The $d-\beta$-Sn structure has been proven to be absent in several III-V/II-VI semiconductors [21], and it is not favoured at any pressure [22]. In addition, Christensen predicted a phase transition for the $r s \rightarrow$ NiAs structure between 30 and $40 \mathrm{GPa}$ [20]. However, no NiAs structure was found within at least $132 \mathrm{GPa}$ by Uehara [23]. The theoretical study by Serrano illustrated that the NiAs structure cannot correspond to a stable high-pressure phase [22]. Christensen[20] stated that anti-NiAs structure cannot be the high-pressure phase of AIN, which agrees with Serrano's research [22]. Thus, the typical phases of AIN (Wz, zb and $r s$ ) were adopted for comparison with the novel proposed phases.

In the present work, we have extensively searched the potential structures of AIN. Four novel high-pressure orthorhombic AIN crystal structures have been predicted. The stabilities of these structures are determined by independent elastic constants and phonon dispersion spectra. We show the results of a total energy study of several phases of AIN. The transition behaviours of various AIN phases under hydrostatic pressure are studied. Further calculations are performed to study the mechanical, thermodynamic and electronic properties of four novel AIN phases.

\section{Computationsl methods}

The crystal structure prediction software CALYPSO (Crystal structure AnaLYsis by Particle Swarm Optimization code) is widely used $[24,25]$ and great achievements in structure prediction have been made with this software [26-29]. The evolutionary simulation method by CALYPSO was performed with none experimental information as guideline. The calculations of geometry optimisations, enthalpies, elastic constants, phonons and band structures were implemented in the CASTEP code [30]. The exchange correlation potential was described by the generalized gradient approximation with Perdew-Burke-Ernzerhof function [31, 32]. 


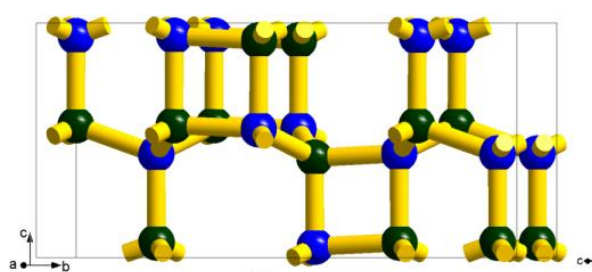

(a)

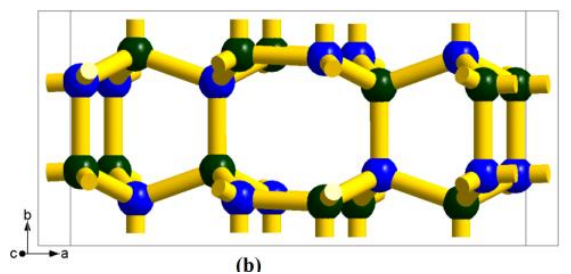

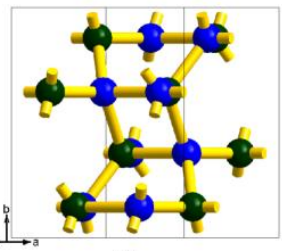

(c)

(d)

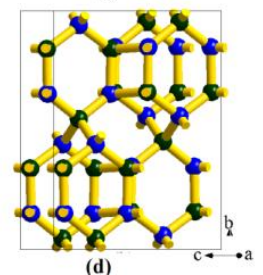

centred $(0,0,0)$ structures with 16 atoms/unit cell. Cmcm-AIN is a C-centred $(0,0,0),(0.5,0.5,0)$ structure with 24 atoms/unit cell. The schematic ambient crystal structures of $P m n 2_{1}$-AIN, Pbam-AIN, Pbca-AIN and Cmcm-AIN are shown in Fig. 1. All the atoms in the four novel structures are composed of coordinates and form $\left[\mathrm{AIN}_{4}\right] /\left[\mathrm{NAl}_{4}\right]$ of tetrahedrons with the four coordination atoms. No $\mathrm{Al}-\mathrm{Al} / \mathrm{N}-\mathrm{N}$ bonds are found in the structures, thereby producing low energy.

Pmn $2_{1}$-AIN (Fig. 1a): In the middle of the Pmn $2_{1}$-AIN unit cell, two similar 'rectangular' structures are located; these non-standard rectangles have four different bond lengths. The two 'rectangles' are both perpendicular to the $a$-axis, one is located at $a=0$ and another is at $a=0.5$.The two terminals of the unit cell are similar to wurtzite structures. The atoms in the 'rectangular' structures and wurtzite structures are equal. Both 'rectangles' are connected by Al$\mathrm{N}$ chemical bonds. In $\mathrm{Pmn} 2_{1}-\mathrm{AIN}$, the bond lengths range from $1.870 \AA$ to $1.934 \AA$.

Pbam-AIN (Fig. 1b): We can divide the 16 atoms into two types ( $\mathrm{A} 1$ and $\mathrm{A} 2$ ) based on the atomic coordinates of the $c$ axis. $\mathrm{A} 1$ atoms are atoms in the surface of the unit cell with $c=0 . A 2$ atoms are atoms inside the unit cell with $c=0.5$. The structure contains three kind rings: four-, six- and eight-membered rings. The planar fourmembered rings and nonplanar eight-membered rings are joined together by non-planar six-membered rings. In the structure of Pbam-AIN, the left part is similar to the right part. Both are two unit cells of $w z$-AIN and connected by the bond parallel to the $a$-axis. Pbam-AlN can be interpreted as a result of crystal restructuring combined with several unit cells of wz-AIN.

Pbca-AIN (Fig. 1c): In the Pbca-AIN unit cell, four parallel atoms layers are present along the $b$-axis. We divide the $b$-axis coordinates of the bottom atom layer as $u$, whereas the other three $b$-axis coordinates are $0.5-u, 0.5+u$ and $1-u$, respectively. In this case, $u$ takes a value of 0.133 . Two kinds of rings are present in the unit cell, one is a four-membered ring and another is a six-membered ring. One atom layer $(0.5-u)$ connects to the other atom layer $(0.5$ $+u$ ) with two parallel Al-N bonds and four-membered ring forms. The four atoms in one atom layer connect with two atoms in the adjacent atom layer and form a non-planar six-membered ring. In each coordination tetrahedron, four Al-N bonds have four different lengths: $1.874,1.879,1.912$ and $1.932 \AA$. All the $\left[\mathrm{AIN}_{4}\right] /\left[\mathrm{NAl}_{4}\right]$ tetrahedrons are not regular tetrahedrons. four novel structures of AIN all belong to the orthorhombic crystal system. Pmn $2_{1}$-AIN, Pbam-AIN and Pbca-AIN are all primitive- 
Table 1 Space number (S.N.), cell parameters $a, b, c(\AA), \rho\left(\mathrm{g} / \mathrm{cm}^{3}\right)$ for four novel AIN phases.

\begin{tabular}{cccccc}
\hline Structure & S.N. & $a$ & $b$ & $c$ & $\rho$ \\
\hline Pmn2 1 & 31 & 3.109 & 10.938 & 5.081 & 3.158 \\
Pbam & 55 & 10.832 & 5.085 & 3.128 & 3.161 \\
Pbca & 61 & 5.265 & 6.191 & 5.416 & 3.085 \\
Cmcm & 63 & 3.179 & 10.398 & 8.190 & 3.017
\end{tabular}

Cmcm-AIN (Fig. 1d): Cmcm-AIN can be viewed to consist of four six-membered rings. The six-membered rings are all perpendicular to the $a$-axis. The rings in the top left corner and bottom right corner share the same coordinates with $a=0.5$. The other two rings share the coordinate $a=0 . \mathrm{Cmcm}$-AIN contains four-membered rings and six-membered rings. In the top (bottom) part of the unit cell, a six-membered ring $(a=0)$ connects with another six-membered ring $(a=0.5)$ via two parallel bonds, whereas the four-membered rings form two parallel bonds and two parallel $b$-axis bonds. The six-membered rings are not standard circles because the bond lengths inside are not equal.

More information on the four AIN novel structures, such as space number, cell parameters $a, b, c$, pare listed in Table 1 and atomic Wyckoff Positions listed in Table 2. After geometric optimisation at ambient pressure, the densities $\left(\rho, \mathrm{g} / \mathrm{cm}^{3}\right)$ of $w z-A I N$, $z b$-AIN and rs-AIN are 3.214, 3.205 and 4.039, respectively. CmcmAIN has the lowest $\rho$, which is $6.13 \%$ lower than that of wz-AIN. Pbca-AIN has a higher $\rho$ than $\mathrm{Cmcm}$-AIN, but its value is lower than any other phase and $4.0 \%$ lower than that of $w z$-AIN. Pmn $2_{1}$-AIN and Pbam-AIN have a similar $\rho$, which are $1.7 \%$ and $1.6 \%$ lower, respectively, than that of $w z-A I N$.

Table 2 Atomic Wyckoff Positions for four novel AIN phases.

\begin{tabular}{cc}
\hline Structure & Atomic Wyckoff Position \\
\hline Pmn $2_{1}$ & Al: $2 a(0.5,0.208,0.066) ;(0.5,0.041,0.565) ;$ \\
& $(0.0,0.286,0.565) ;(0.0,0.463,0.926)$ \\
& N: $2 a(0.5,0.538,0.048) ;(0.5,0.713,0.445) ;$ \\
& $(0.5,0.208,0.442) ;(0.5,0.041,0.943)$ \\
$P b a m$ & Al: $4 h(0.834,0.180,0.5) ; 4 g(0.912,0.682,0)$ \\
& N: $4 h(0.333,0.696,0.5) ; 4 g(0.413,0.199,0)$ \\
Pbca & Al: $8 c(0.177,0.633,0.912) ;$ \\
& N: $8 c(0.690,0.367,0.408)$ \\
$C m c m$ & Al: $4 c(0.5,0.547,0.75) ; 8 f(0.0,0.340,0.941)$ \\
& N: $4 c(0.0,0.440,0.75) ; 8 f(0.5,0.348,0.436)$
\end{tabular}

Table $3 C_{i j}(\mathrm{GPa})$ for four novel AIN phases.

\begin{tabular}{lcccc}
\hline & Pmn2 $_{1}$ & Pbam & Pbca & Cmcm \\
\hline$C_{11}$ & 381.9 & 347.7 & 252.1 & 339.1 \\
$C_{22}$ & 345.9 & 290.7 & 309.8 & 277.7 \\
$C_{33}$ & 287.9 & 381.5 & 376.0 & 291.7 \\
$C_{44}$ & 114.5 & 112.1 & 104.2 & 131.6 \\
$C_{55}$ & 111.8 & 113.0 & 110.2 & 87.4 \\
$C_{66}$ & 113.2 & 111.7 & 116.1 & 107.9 \\
$C_{12}$ & 126.0 & 98.7 & 117.9 & 81.4 \\
$C_{13}$ & 98.3 & 128.1 & 102.1 & 123.1 \\
$C_{23}$ & 100.6 & 96.8 & 124.1 & 123.1 \\
\hline
\end{tabular}

\section{Stability Analysis}

To analyse the mechanical stability and dynamical stability of the novel high-pressure phases of AIN, the elastic constants and phonon dispersion spectra are studied.

For an orthorhombic crystal system, the mechanical stability can be evaluated by [35].

$C_{i i}>0 ;(i=1,2,3,4,5,6) ;\left(C_{11}+C_{22}-2 C_{12}\right)>0$;

$\left(C_{11}+C_{33}-2 C_{13}\right)>0 ;\left(C_{22}+C_{33}-2 C_{23}\right)>0$;

$\left[C_{11}+C_{22}+C_{33}+2\left(C_{12}+C_{13}+C_{23}\right)\right]>0$
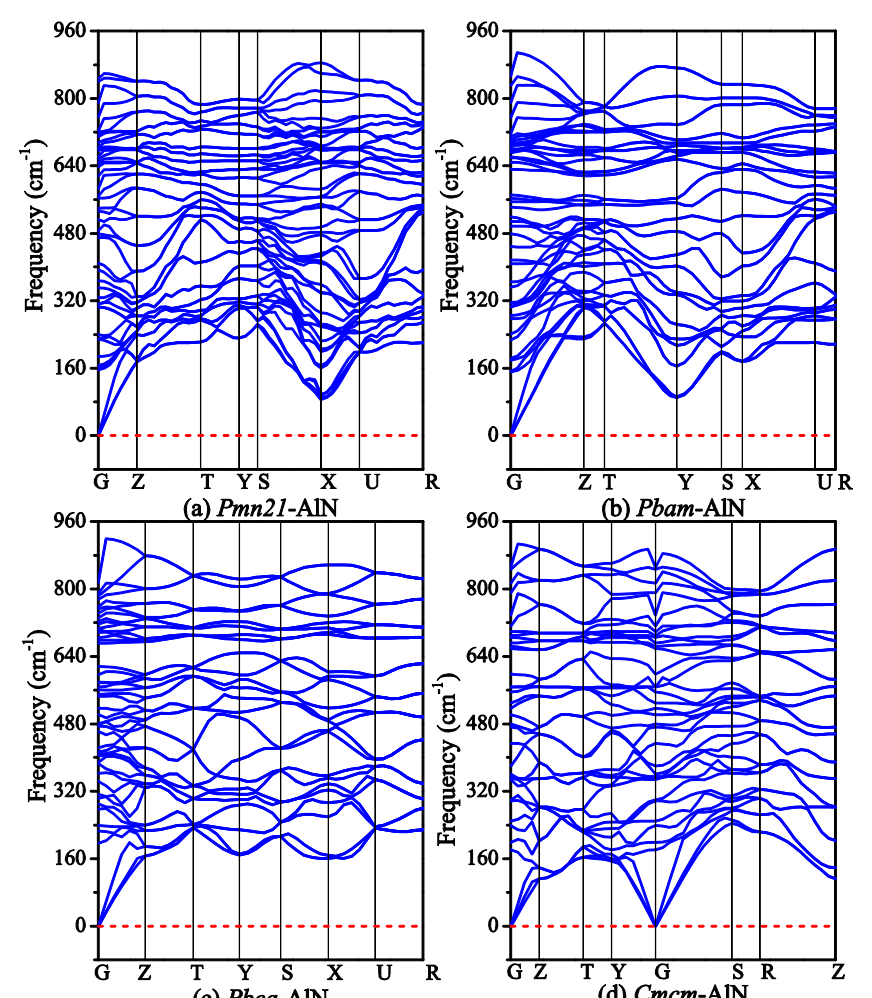

(c) Pbca-AlN

(d) $\mathrm{Cmcm}$-AIN

Fig. 2. Phonon dispersion spectra for the four novel AIN phases. 
According to the abovementioned criteria, the independent elastic constants $\left(C_{i j}\right)$ listed in Table 3 show that $P m n 2_{1}$-AIN, PbamAIN, Pbca-AIN and Cmcm-AIN are all mechanically stable under ambient pressure.

The calculated phonon dispersion spectra for $\mathrm{Pmn} 2_{1}$-AIN, PbamAIN, Pbca-AIN and Cmcm-AIN are shown in Fig. 2. No imaginary frequency is observed during the whole Brillouin zone. All four structures are dynamically stable.

\section{Phase Transformation under Pressure}

As a fundamental thermodynamic variable, pressure has a significant impact on the chemical and physical properties of a material. High-pressure technology is an interesting field in materials research.

The relative enthalpies for the chosen structures are presented in Fig. 3 and compared with those of $w z-A I N$ as a function of pressure up to $20 \mathrm{GPa}$. Wz-AIN is more favourable than any other AIN phase at ambient pressure. Zb-AIN has a constant relative enthalpy (only approximately $0.05 \mathrm{eV}$ per formula unit (f.u.) higher than that of $w z-A I N)$ within the pressure range studied. At ambient pressure, the enthalpies of $P m n 2_{1}$-AIN, Pbam-AIN, Pbca-AIN and $\mathrm{Cmcm}$-AIN are more favoured than that of $\mathrm{rs}$-AIN, ranging from the smallest value of $0.150 \mathrm{eV} / \mathrm{f} . \mathrm{u}$. (Cmcm-AIN) to the largest value of $0.305 \mathrm{eV} / \mathrm{f} . u$. (Pmn2 ${ }_{1}$-AIN). When the pressure exceeds $4.34 \mathrm{GPa}$, the rs-AIN favours enthalpy more than $\mathrm{Cmcm}$-AIN. As the pressure increases to $9.07 \mathrm{GPa}$, rs-AIN favours enthalpy more than Pbca-AIN. Finally, when the pressure reaches $11.14 \mathrm{GPa}$, all four novel phases lose their enthalpy advantage over rs-AIN.

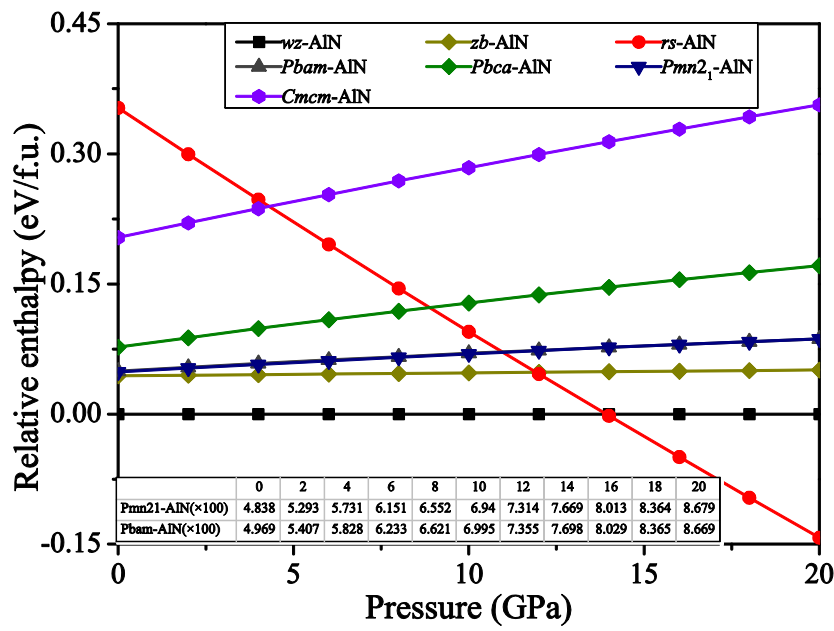

Fig. 3. Calculated enthalpy curves (relative to $w z-A I N)$ as a function of pressure for various AIN phases.
Wz-AIN clearly transforms to rs-AIN under a hydrostatic pressure of $14 \mathrm{GPa}$, which is in good agreement with the previous experiment [13-16], and other theoretical studies [36-38]. Thus, all AIN phases undergo transformation to $r s$-AIN as the hydrostatic pressure reaches $14 \mathrm{GPa}$ in terms of enthalpy.

The enthalpies-pressure relationship indicates that the four AIN phases are not pressure driven. Therefore, these phases might not be achieved via a high-pressure approach during loading pressure. The formation of the metastable phases is affected by the decompression rate. For example, the Si-XII (R8), Si-III (BC8) and GeIII (ST12) phases can be acquired by releasing pressure from the $\beta$ Sn phases of Si and Ge (Si-II or Ge-II) slowly [39, 40]. However, rapid depressurisation causes the formation of the Ge-IV (BC8) phase and the tetragonal Si-VIII/IX phases [41, 42]. Similarly, the four novel AIN phases may be achieved by adjusting the pressure release rate.

\section{Mechanical Properties}

The pressure-volume relationships for four crystal structures of AIN are deduced by fitting the third-order Birth-Murnaghan equation of state (EOS) as shown in Fig. 4 [43].

$P(V)=\frac{3}{2} B_{0}\left[\left(\frac{V}{V_{0}}\right)^{-\frac{7}{3}}-\left(\frac{V}{V_{0}}\right)^{-\frac{5}{3}}\right]\left\{1+\frac{3}{4}\left(B_{0}^{\prime}-4\right)\left[\left(\frac{V}{V_{0}}\right)^{-\frac{2}{3}}-1\right]\right\}$

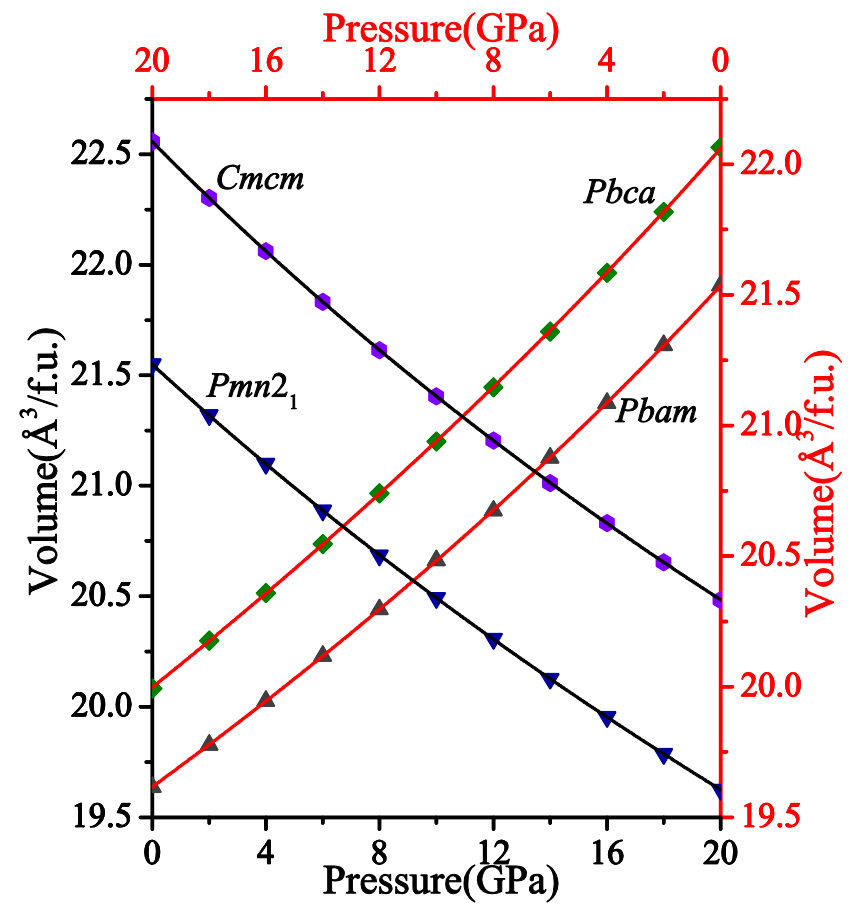

Fig. 4. Volume of the four AIN phases as a function of pressure. The solid geometric pattern and solid lines represent the calculated data and fitting results, respectively. 
Where $\boldsymbol{V}_{\mathbf{0}}$ is the volume per f.u. at ambient pressure; $\mathrm{V}$ is the volume per f.u. at pressure $P ; \boldsymbol{B}_{\mathbf{0}}$ is the isothermal bulk modulus; $\boldsymbol{B}_{\mathbf{0}}^{\prime}$ is the first order pressure derivative of the isothermal bulk modulus. The values of $\boldsymbol{B}_{\mathbf{0}}, \boldsymbol{B}_{\mathbf{0}}^{\prime}$, and $\boldsymbol{V}_{\mathbf{0}}$ are obtained from Equation (2) and listed in Table 4; these values agree well with previous reports $[22,37,44]$.

The volume decreases with increasing pressure for all the proposed phases. Those four phases have similar volume compressibility ( 9\%), thereby suggesting their good anticompressibility. The volume compressibility is different depending on the various phases. Pbca-AIN has the largest volume compressibility (9.39\%), whereas Pbam-AIN has the smallest (8.92\%) among all four novel phases at the pressure range of 0-20 GPa.

For the orthorhombic crystal system, the Voigt form and the Reuss form of bulk modulus $(B)$ and shear modulus $(G)$ can be obtained from the independent elastic constants $\left(C_{i j}\right)$ [45].

Based on the Voigt-Reuss-Hill relationship [46], the Hill form $B$ $(G)$ are arithmetic mean values of the Voigt form $B(G)$ and Reuss form $B(G)$. Young's modulus $(E)$ and Poisson's ratio $(\sigma)$ are calculated from $B$ and $G$ as follows:

$E=9 B G /(3 B+G) ; \sigma=(3 B-2 G) /(6 B+2 G)$

The values of $B, G, E, \sigma$ and $B / G$ are presented in Table 4 . In generally, $B$ represents the resistance to volume change by load pressure, $G$ represents the resistance to reversible deformations upon shear stress [47]. The higher value of $E$, the stiffer the material. All the novel phases of AIN have large $B, G$ and $E$, which indicate their potential applications as hard materials for cutting and machining.

Table $4 B_{0}(\mathrm{GPa}), V_{0}\left(\AA^{3} / \mathrm{f} . u\right.$.), $B_{0}^{\prime}, B(\mathrm{GPa}), G(\mathrm{GPa}), E(\mathrm{GPa}), \sigma, G / B$ ratio and $H v(\mathrm{GPa})$ for the four novel AIN phases at ambient pressure.

\begin{tabular}{lcccc}
\hline & Pmn2 $_{1}$ & Pbam & Pbca & Cmcm \\
\hline$B_{0}$ & 182.7 & 183.2 & 178.8 & 172.7 \\
$V_{0}$ & 21.5 & 21.5 & 22.1 & 22.6 \\
$B_{0}^{\prime}$ & 3.40 & 3.38 & 2.74 & 3.81 \\
$B$ & 183.2 & 183.4 & 178.2 & 173.1 \\
$G$ & 113.6 & 113.5 & 104.5 & 102.2 \\
$E$ & 282.4 & 282.3 & 262.2 & 256.2 \\
$\sigma$ & 0.24 & 0.24 & 0.25 & 0.25 \\
$G / B$ & 0.62 & 0.62 & 0.59 & 0.59 \\
$H v$ & 15.2 & 15.2 & 13.3 & 13.2 \\
\hline
\end{tabular}

The fitted $B$ values from the third-order Birth-Murnaghan EOS are almost equal to the values calculated from the independent elastic constants, thereby confirming the reliability of our calculations.

Hardness is used as one of the fundamental mechanical properties of materials. We further analysed the Vickers hardness $(H v)$ of different phases by adopting Chen's formula based on empirical scheme [48]:

$H_{V}=2\left(\kappa^{2} G\right)^{0.585}-3 ; \kappa=G / B$

The obtained values of $H v$ according to Equation (4) are also presented in Table 4. The hardness of all the proposed phases range from 13.2 GPa to $15.2 \mathrm{GPa}$, which enrich the hardness of AIN compounds and may extend the industrial application of AIN.

\section{Electronic Properties}

The calculated band structures of the four novel phases of AIN along the selected high symmetry directions of the first Brillouin zone at ambient pressure are shown in Fig. 5. All the AIN phases possess semiconductive properties that can be attributed to the direct band gaps at the $G$ points. For the four novel structures of AIN, the band gaps are 3.889 (Pmn2 ${ }_{1}$-AIN), 3.927 (Pbam-AIN), 3.855 (Pbca-AIN) and $3.627 \mathrm{eV}$ (Cmcm-AIN). Given the wide direct band gaps, these four novel phases AIN may be used as semiconductor devices and optoelectronic devices.
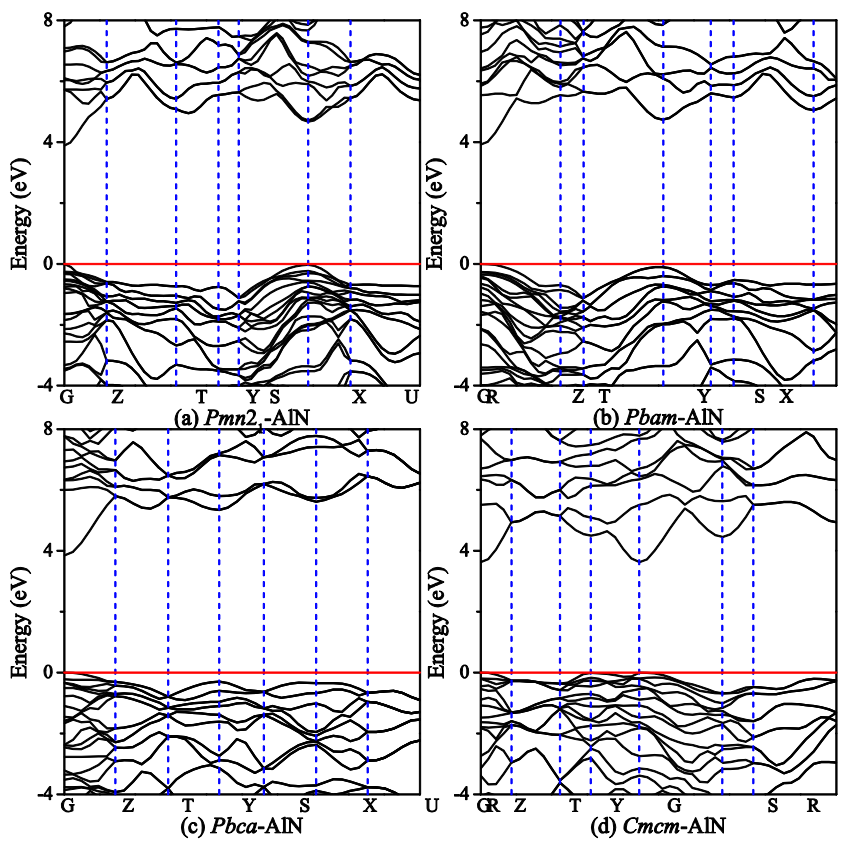

Fig. 5. Calculated band structures for the four novel AIN phases at ambient pressure. The Fermi level is represented by a horizontal red line. 


\section{Conclusion}

In this work, four novel orthorhombic AIN phases are proposed after using an evolutionary methodology. Based on first-principles calculations, the enthalpy of all four phases $\left(\mathrm{Pmn}_{1}{ }_{1}\right.$-AIN, Pbam-AIN, Pbca-AIN and Cmcm-AIN) will be lower than that of $r s$-AIN at ambient pressure. The calculated elastic constants and phonon dispersion spectra certify the mechanically and dynamically stabilities of the four novel phases. The calculated $\mathrm{Hv}$ is based on an empirical relation; the resulting values suggest all the four novel AIN phases possess a high hardness range from $13.2 \mathrm{GPa}$ to $15.2 \mathrm{GPa}$. The electronic band structure calculations suggest that all the AIN phases possess semiconductive properties with wide direct band gaps. The superior mechanical properties and adjustable band gaps of these phases indicate that these metastable phases of AIN may have potential industrial applications in the future.

\section{Acknowledgements}

This work was supported by the National Science Foundation of China (Grant Nos. 51421091 and 51332005) and the Postgraduate Innovation Project of Hebei Province of China (Grant No. 00302-6370007).

\section{References}

[1] G.A. Slack, R.A. Tanzilli, R. Pohl, J. Vandersande, J. Phys. Chem. Solids, 48 (1987) 641-647.

[2] K. Khor, K. Cheng, L. Yu, F. Boey, Materials Science and Engineering: A, 347 (2003) 300-305.

[3] J.A. Dean, McGraw-Hill, Inc, 4 (1999).

[4] M. Kazan, E. Moussaed, R. Nader, P. Masri, Physica Status Solidi C, 4 (2007) 204-207.

[5] Y.Ö. Çiftci, K. Çolakoğlu, E. Deligöz, physica status solidi (c), 4 (2007) 234-237.

[6] A.J. Wang, S.L. Shang, Y. Du, Y. Kong, L.J. Zhang, L. Chen, D.D. Zhao, Z.K. Liu, Comput. Mater. Sci., 48 (2010) 705-709.

[7] I. Yonenaga, T. Shima, M.H. Sluiter, Jpn. J. Appl. Phys., 41 (2002) 4620-4621.

[8] B.D. Fulcher, X.Y. Cui, B. Delley, C. Stampfl, Phys. Rev. B, 85 (2012) 184106.

[9] M. Schwarz, M. Antlauf, S. Schmerler, K. Keller, T. Schlothauer J. Kortus, G. Heide, E. Kroke, High. Pres. Res., 34 (2013) 1-17.

[10] L.M. Svedberg, K.C. Arndt, M.J. Cima, J. Am. Ceram. Soc., 83 (2000) 41-46.
[11] J. Li, K.B. Nam, M.L. Nakarmi, J.Y. Lin, H.X. Jiang, P. Carrier, S.-H. Wei, Appl. Phys. Lett., 83 (2003) 5163.

[12] I. Petrov, E. Mojab, R.C. Powell, J.E. Greene, L. Hultman, J.E. Sundgren, Appl. Phys. Lett., 60 (1992) 2491.

[13] H. VOLLSTÄDT, E. ITO, M. AKAISHI, S.-i. AKIMOTO, O. FUKUNAGA, Proc. Japan Acad. B, 66 (1990) 7-9.

[14] Q. Xia, H. Xia, A.L. Ruoff, J. Appl. Phys., 73 (1993) 8198-8200.

[15] I. Gorczyca, N. Christensen, P. Perlin, I. Grzegory, J. Jun, M. Bockowski, Solid State Commun., 79 (1991) 1033-1034.

[16] Z. Wang, K. Tait, Y. Zhao, D. Schiferl, C. Zha, H. Uchida, R.T. Downs, J. Phys. Chem. B, 108 (2004) 11506-11508.

[17] R. Nelmes, M. McMahon, Semiconductors and semimetals, 54 (1998) 145-246.

[18] G.J. Ackland, Rep. Prog. Phys., (2001).

[19] J. Van Vechten, Phys. Rev. B, 7 (1973) 1479.

[20] N.E. Christensen, I.I. Gorczyca, Phys Rev B Condens Matter, 47 (1993) 4307-4314.

[21] R. Nelmes, M. McMahon, S. Belmonte, Phys. Rev. Lett., 79 (1997) 3668.

[22] J. Serrano, A. Rubio, E. Hernández, A. Muñoz, A. Mujica, Phys. Rev. B, 62 (2000) 16612.

[23] S. Uehara, T. Masamoto, A. Onodera, M. Ueno, O. Shimomura, K. Takemura, J. Phys. Chem. Solids, 58 (1997) 2093-2099.

[24] Y.C. Wang, J.A. Lv, L. Zhu, Y.M. Ma, Phys. Rev. B, 82 (2010) 094116.

[25] Y.C. Wang, J. Lv, L. Zhu, Y.M. Ma, Comput. Phys. Commun., 183 (2012) 2063-2070.

[26] M. Hu, F. Tian, Z.S. Zhao, Q. Huang, B. Xu, L.M. Wang, H.T. Wang, Y.J. Tian, J.L. He, Journal Of Physical Chemistry C, 116 (2012) 24233-24238.

[27] Z. Zhao, B. Xu, L.M. Wang, X.F. Zhou, J. He, Z. Liu, H.T. Wang, Y. Tian, ACS Nano, 5 (2011) 7226-7234.

[28] M. Zhang, H. Liu, Q. Li, B. Gao, Y. Wang, H. Li, C. Chen, Y. Ma, Phys. Rev. Lett., 114 (2015) 015502.

[29] H. Liu, Y. Yao, D.D. Klug, Phys. Rev. B, 91 (2015) 014102.

[30] S.J. Clark, M.D. Segall, C.J. Pickard, P.J. Hasnip, M.J. Probert, K. Refson, M.C. Payne, Z. Kristallogr., 220 (2005) 567-570.

[31] J.P. Perdew, K. Burke, M. Ernzerhof, Phys. Rev. Lett., 77 (1996) 3865-3868.

[32] W. Kohn, L.J. Sham, Phys. Rev., 140 (1965) A1133-A1138.

[33] D. Vanderbilt, Phys Rev B Condens Matter, 41 (1990) 78927895.

[34] H.J. Monkhorst, J.D. Pack, Phys. Rev. B, 13 (1976) 5188. 
[35] J.F. Nye, Physical Properties of Crystals: Their Representation by Tensors and Matrices, Oxford University Press, 1985.

[36] M. Durandurdu, J. Alloys Compd., 480 (2009) 917-921.

[37] F. Peng, D. Chen, H.Z. Fu, X.L. Cheng, Physica B Condensed Matter, 403 (2008) 4259-4263.

[38] S. Louhibi-Fasla, H. Achour, K. Kefif, Y. Ghalem, Physics Procedia, 55 (2014) 324-328.

[39] J. Crain, G. Ackland, J. Maclean, R. Piltz, P. Hatton, G. Pawley, Phys. Rev. B, 50 (1994) 13043.

[40] C.S. Menoni, J.Z. Hu, I.L. Spain, Phys. Rev. B, 34 (1986) 362.

[41] Y.-X. Zhao, F. Buehler, J.R. Sites, I.L. Spain, Solid State Commun., 59 (1986) 679-682.

[42] R. Nelmes, M. McMahon, N. Wright, D. Allan, J. Loveday, Phys. Rev. B, 48 (1993) 9883.

[43] F. Birch, J. Appl. Phys., 9 (1938) 279-288.

[44] U.P. Verma, P.S. Bisht, Solid State Sci., 12 (2010) 665-669.

[45] J.P. Watt, J. Appl. Phys., 50 (1980) 6290-6295.

[46] R. Hill, Proc. Phys. Soc. A, 65 (1952) 349-354.

[47] H.B. Ozisik, K. Colakoglu, E. Deligoz, H. Ozisik, J. Mol. Model., 18 (2012) 3101-3112.

[48] X.Q. Chen, H.Y. Niu, D.Z. Li, Y.Y. Li, Intermetallics, 19 (2011) $1275-1281$. 